\title{
Properties of Common Focus Point Gathers
}

\author{
Jan Thorbecke and Scott Morton \\ Cray Research / Silicon Graphics, \\ Petroleum Applications, \\ 655E Lone Oak Drive, Eagan, \\ Minnesota 55121, USA
}

April 7, 1997 


\section{Introduction}

Common Focus Point technology, as described by Berkhout (1997) and Thorbecke (1997), is a new approach to the processing of seismic data. The Common Focus Point (CFP) gather is a migration gather suitable for a variety of seismic processes including velocity analysis, focusing operator updating and AVO analysis. In this paper, we will discuss the construction of CFP gathers and describe several applications of the CFP technology.

\section{Construction of a CFP gather}

A CFP-gather is constructed from shot records, requiring an initial focusing operator which can be derived from stacking velocities or some macro model. In the latter case, the operator is calculated by positioning a point source at the focal point location followed by a forward modeling experiment to calculate the response at the surface; the operator represents the Green's function of the focus point in the macro model. Using this initial focusing operator, a CFP gather for focusing in detection is constructed by a time-domain convolution between the traces of the focusing operator and the traces in the shot record, followed by an integration over all the receivers present in the shot record. This process is equivalent to a non-recursive wavefield extrapolation of the shot record to a single focus point in the subsurface and results in a single trace in the CFP gather.

This process is illustrated in figure 1 , for the focus point defined at a synclinal interface and indicated with a white bullet in figure 1a). The time-reversed focusing operator (figure 1b) is applied to all shot records available; three shot records with source positions at $x=-495, x=0$ and $x=495 \mathrm{~m}$ are shown in figures $1 \mathrm{c}, \mathrm{d}$ and e respectively. Convolution along the time axis of each of the traces in the shot records with the corresponding traces in the focusing operator gives the intermediate records shown in figures $1 \mathrm{f}, \mathrm{g}$ and $\mathrm{h}$. Summation over all the traces in the intermediate records yields one trace of the CFP gather posted at the source position of the original shot record. The most important contribution in the CFP-gather trace, the focus point response (see 1i), is from the Fresnel zone of the focus point.

\section{Properties of CFP gathers}

The CFP gather has the following characteristics:

- If the correct operator is used, the traveltimes of the focus point response in the CFP gather are equal to the traveltimes of the focusing operator, the so-called 'principle of equal traveltimes' (Berkhout, 1997).

- However, an error in the operator results in an opposing error in the CFP gather. Hence the difference between the operator and the focus point response can be used to correct the operator. This feature of CFP technology was applied by Morton and Thorbecke (1996) to show that operator updating in the CFP domain is simple enough to be carried out automatically.

- The amplitude of the focus point response is directly related to the angle-dependent reflection coefficient of the reflector at the defined focus point. The AVO behavior of the focus point can be extracted from the CFP gather by a stabilized division of the CFP gather by the (correct) focusing operator.

- A structural image for the neighborhood of the focus point is obtained by using the focusing operator to stack the CFP gather. Consequently, a Kirchhoff implementation of the CFP technology can simultaneously perform operator updating and target-oriented imaging with computational effort comparable to that of a single Kirchhoff migration of the target. So the CFP imaging procedure can be considered as a conventional Kirchhoff migration broken up into two separate steps. 

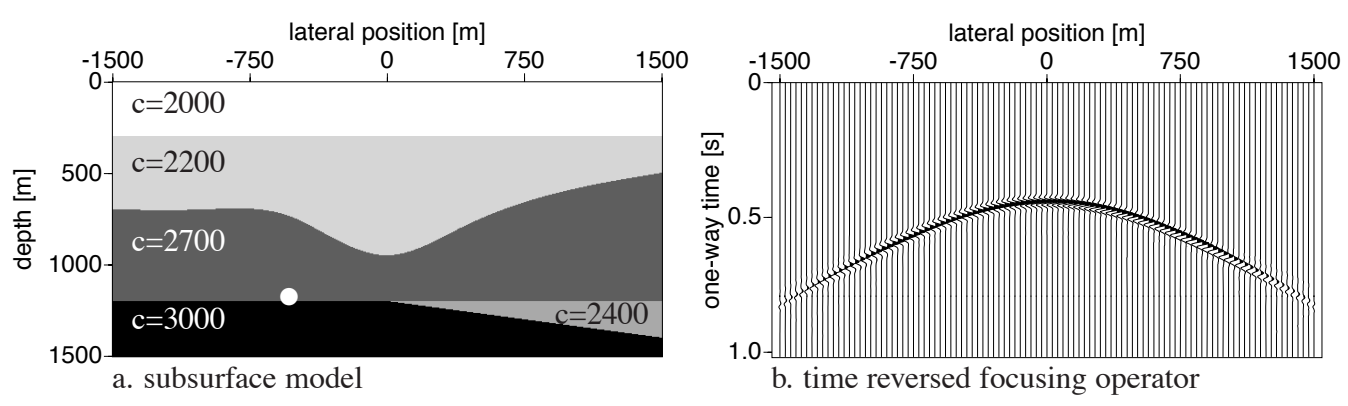

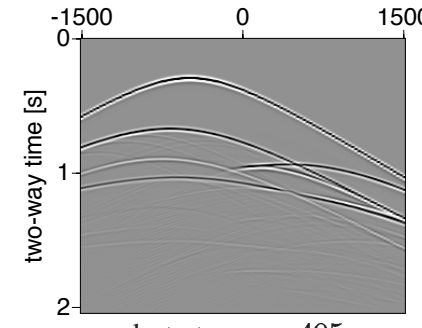

c. shot at $x=-495$
$1500-1500 \quad 0$

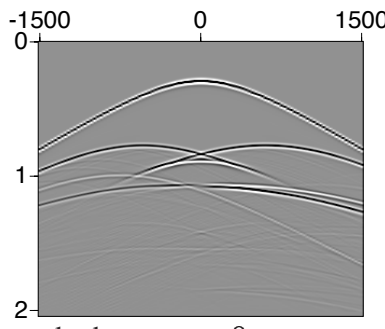

d. shot at $x=0$

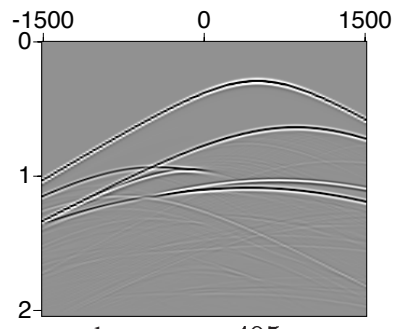

e. shot at $x=495$

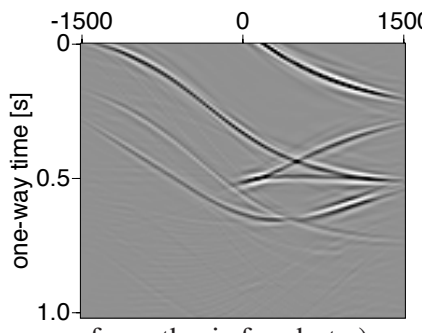

f. synthesis for shot c)

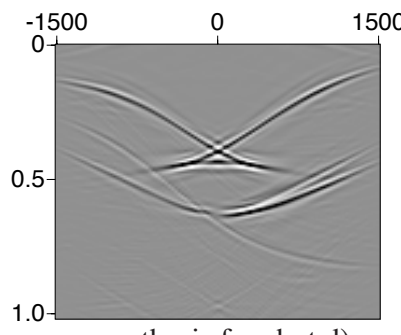

g. synthesis for shot d)

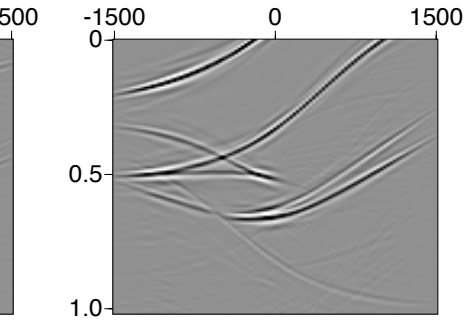

h. synthesis for shot e)

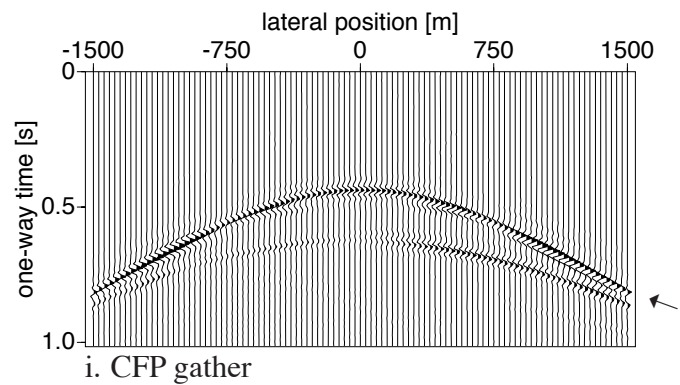

Figure 1: Construction of a CFP gather for focusing in detection. Every shot record contributes to one trace, positioned at the source position, in the CFP gather. The contribution of the Fresnel zones, the region around the extrema in figures 1 , $1 \mathrm{~g}$ and $1 \mathrm{~h}$, are clearly visible on the CFP gather in figure 1i. The focus point response has been indicated with an arrow. Note that the bow-tie event in the shot records is focused in the CFP gather to a single event.

\section{Summary}

In this abstract, we have presented the method of construction of common focus point (CFP) gathers and described several applications of the CFP technology. One of the most important applications is the ability to estimate correct focusing operators without requiring an updating of the macro model. In the presentation, the CFP approach will be illustrated using synthetic and field data.

\section{References}

Berkhout, A. J., 1997, Pushing the limits of seismic imaging: part i: Geophysics, 62, in print.

Morton, S., and Thorbecke, J. W., 1996, Automating prestack migration analysis using common focal point gathers: 65th Ann. Internat. Mtg., SEG, Expanded Abstracts, 1126-1129.

Thorbecke, J. W., 1997, Common focus point technology: Ph.D. thesis, Delft University of Technology. 